Pacific

Journal of

Mathematics

HOLOMORPHY OF IGUSA'S AND TOPOLOGICAL ZETA FUNCTIONS FOR HOMOGENEOUS POLYNOMIALS

B. Rodrigues ANd W. Veys 


\title{
HOLOMORPHY OF IGUSA'S AND TOPOLOGICAL ZETA FUNCTIONS FOR HOMOGENEOUS POLYNOMIALS
}

\author{
B. Rodrigues And W. Veys
}

Let $F$ be a number field and $f \in F\left[x_{1}, \ldots, x_{n}\right] \backslash F$. To any completion $K$ of $F$ and any character $\kappa$ of the group of units of the valuation ring of $K$ one associates Igusa's local zeta function $Z^{K}(\kappa, f, s)$. The holomorphy conjecture states that for all except a finite number of completions $K$ of $F$ we have that if the order of $\kappa$ does not divide the order of any eigenvalue of the local monodromy of $f$ at any complex point of $f^{-1}\{0\}$, then $Z^{K}(\kappa, f, s)$ is holomorphic on $\mathbb{C}$. The second author already showed that this conjecture is true for curves, i.e., for $n=2$. Here we look at the case of an homogeneous polynomial $f$, so we can consider $\{f=0\} \subseteq \mathbb{P}^{n-1}$. Under the condition that $\chi\left(\mathbb{P}_{\mathbb{C}}^{n-1} \backslash\{f=0\}\right) \neq 0$ we prove the holomorphy conjecture. Together with some results in the case when $\chi\left(\mathbb{P}_{\mathbb{C}}^{n-1} \backslash\{f=0\}\right)=0$, we can conclude that the holomorphy conjecture is true for an arbitrary homogeneous polynomial in three variables.

We also prove the so-called monodromy conjecture for a homogeneous polynomial $f \in F\left[x_{1}, x_{2}, x_{3}\right]$ with $\chi\left(\mathbb{P}_{\mathbb{C}}^{2} \backslash\{f=\right.$ $0\}) \neq 0$.

\section{Introduction.}

0.1. Let $K$ be a finite extension of the field $\mathbb{Q}_{p}$ of $p$-adic numbers, $R_{K}$ the valuation ring of $K, P_{K}$ the maximal ideal of $R_{K}, \pi$ a fixed uniformizing parameter for $R_{K}$, and $\bar{K}=R_{K} / P_{K}$ the residue field of $K$ with cardinality $q$. For $z \in K, \operatorname{ord}_{\pi} z \in \mathbb{Z} \cup\{+\infty\}$ denotes the valuation of $z,|z|=q^{-\operatorname{ord}_{\pi} z}$ and $\operatorname{ac}(z)=z \pi^{-\operatorname{ord}_{\pi} z}$ is the angular component of $z$.

Let $f(x) \in K[x], x=\left(x_{1}, \ldots, x_{n}\right)$, be a nonconstant polynomial and $\kappa$ a character of $R_{K}^{\times}$, i.e., a homomorphism $\kappa: R_{K}^{\times} \rightarrow \mathbb{C}^{\times}$with finite image, where $R_{K}^{\times}$denotes the group of units of $R_{K}$. (We formally put $\kappa(0)=0$.) To these data one associates Igusa's local zeta function $Z^{K}(\kappa, f, s)$, which is the meromorphic continuation to $\mathbb{C}$ of

$$
s \longmapsto \int_{R_{K}^{n}} \kappa(a c f(x))|f(x)|^{s}|d x|,
$$


for $\Re(s)>0$, where $|d x|$ denotes the Haar measure on $K^{n}$, normalized such that $R_{K}^{n}$ has measure 1 . Replacing $R_{K}^{n}$ by $P_{K}^{n}$ we analogously define $Z_{0}^{K}(\kappa, f, s)$. Igusa [7] showed that they are rational functions of $q^{-s}$.

0.2. We can write $Z^{K}(\kappa, f, s)$ and $Z_{0}^{K}(\kappa, f, s)$ in terms of an embedded resolution $h^{K}: Y^{K} \rightarrow \mathbb{A}^{n}(K)$ of $f^{-1}\{0\}$ in $\mathbb{A}^{n}(K)$, see Theorem 1.5.1. Let $E_{j}^{K}, j \in T^{K}$, be the (reduced) irreducible components of $\left(h^{K}\right)^{-1}\left(f^{-1}\{0\}\right)$, and let $N_{j}$ be the multiplicity of $E_{j}^{K}$ in the divisor of $f \circ h^{K}$ on $Y^{K}$. Then Theorem 1.5.1 implies that when the order of $\kappa$ divides no $N_{j}$ at all, the zeta functions $Z^{K}(\kappa, f, s)$ and $Z_{0}^{K}(\kappa, f, s)$ will be holomorphic on $\mathbb{C}$. Now the $N_{j}$ are not intrinsically associated to $f^{-1}\{0\}$; but the order (as root of unity) of any eigenvalue of the local monodromy on $f^{-1}\{0\}$ divides some $N_{j}$, and those eigenvalues are intrinsic invariants of $f^{-1}\{0\}$ (see (1.6)). This observation inspired Denef [2, Conjecture 4.4.2] to propose the following.

0.3. Holomorphy Conjecture. Let $f \in F\left[x_{1}, \ldots, x_{n}\right] \backslash F$ for some number field $F$. Then for almost all completions $K$ of $F$ (i.e., for all except a finite number) we have the following for any character $\kappa$ of $R_{K}^{\times}$. If the order of $\kappa$ does not divide the order of any eigenvalue of the (complex) local monodromy of $f$ at any complex point of $f^{-1}\{0\}$, then $Z^{K}(\kappa, f, s)$ is holomorphic on $\mathbb{C}$.

0.4. Remark. Denef also formulated this conjecture for $Z_{0}^{K}(\kappa, f, s)$ and for a generalization of those two involving a Schwartz-Bruhat function, i.e., a locally constant function with compact support.

0.5. The second author showed in $[\mathbf{1 0}]$ that this conjecture is true for curves, i.e., for $f \in F\left[x_{1}, x_{2}\right]$. In this paper we consider the case of a homogeneous polynomial $f$, but in an arbitrary number of variables; thus $f \in F\left[x_{1}, \ldots, x_{n}\right]$. Remark that for such $f$ we can consider $\{f=0\} \subseteq$ $\mathbb{P}_{\mathbb{C}}^{n-1}$. Under the condition that $\chi\left(\mathbb{P}_{\mathbb{C}}^{n-1} \backslash\{f=0\}\right) \neq 0$ we will prove the holomorphy conjecture for $f$. (Here $\chi(\cdot)$ denotes the topological EulerPoincaré characteristic.) If this condition is not fulfilled we will formulate a sort of 'projective holomorphy conjecture' implying Conjecture 0.3 as we will prove in Theorem 3.5. Important is that with this projective version of the holomorphy conjecture we actually drop the dimension by one. This will enable us to prove the holomorphy conjecture for an arbitrary homogeneous polynomial in three variables by reducing the problem to the situation of curves. We will also prove all these results for the so-called topological zeta function (see (2.3) for the definition of this function).

In the last section we will prove the monodromy conjecture for a homogeneous polynomial $f$ in three variables (under the condition that $\chi\left(\mathbb{P}_{\mathbb{C}}^{2} \backslash\{f=\right.$ $0\}) \neq 0)$. This conjecture roughly states that if $s_{0}$ is a pole of $Z^{K}(\kappa, f, s)$, then $\exp \left(2 \pi \sqrt{-1} \Re\left(s_{0}\right)\right)$ is an eigenvalue of the local monodromy of $f$ at some complex point of $f^{-1}\{0\}$. 


\section{Explicit formulas.}

1.1. In this section we will construct some embedded resolutions playing the key role in the proof of our results. We will also state some general formulas for Igusa's local zeta function and for eigenvalues of the local monodromy of $f$ in terms of those embedded resolutions.

1.2. Let $f \in F\left[x_{1}, \ldots, x_{n}\right] \backslash F$ be a homogeneous polynomial over some field $F$ of characteristic zero, $\mathcal{P}=\operatorname{Proj} F\left[x_{1}, \ldots, x_{n}\right]$ and $\mathcal{D}=\operatorname{Proj}\left(F\left[x_{1}, \ldots\right.\right.$, $\left.x_{n}\right] /(f)$ ). An embedded resolution of $\mathcal{D}$ in $\mathcal{P}$ consists of a nonsingular variety $\mathcal{Y}$ and a proper birational morphism $\varphi: \mathcal{Y} \rightarrow \mathcal{P}$ such that the restriction $\varphi: \mathcal{Y} \backslash \varphi^{-1}(\mathcal{D}) \longrightarrow \mathcal{P} \backslash \mathcal{D}$ is an isomorphism and $\varphi^{-1}(\mathcal{D})$ has normal crossings in $\mathcal{Y}$. By Hironaka [6] we can choose such an embedded resolution $(\mathcal{Y}, \varphi)$ of $\mathcal{D}$ in $\mathcal{P}$ over $F$ by means of blowing-ups. Let $\mathcal{E}_{i}, i \in \mathcal{T}=\mathcal{T}_{e} \cup \mathcal{T}_{s}$, denote the (reduced) $F$-irreducible components of $\varphi^{-1}(\mathcal{D})$, where $i \in \mathcal{T}_{s}$ if and only if $\mathcal{E}_{i}$ is a (reduced) irreducible component of the strict transform of $\mathcal{D}$.

Then we can write $f=\prod_{i \in \mathcal{T}_{s}} f_{i}^{N_{i}}$, where each $f_{i}$ is an irreducible homogeneous polynomial over $F$ and $f_{i}$ corresponds with $\mathcal{E}_{i}\left(i \in \mathcal{T}_{s}\right)$ in the obvious way. Let $P$ be the divisor of $f$ on $\mathcal{P}$, i.e., $P=\operatorname{div} f=\sum_{i \in \mathcal{T}_{s}} N_{i} P_{i}$, with $P_{i}=\operatorname{Proj}\left(F\left[x_{1}, \ldots, x_{n}\right] /\left(f_{i}\right)\right)$. Then for $i \in \mathcal{T}$ we define $N_{i}$ to be the multiplicity of $\mathcal{E}_{i}$ in the divisor $\varphi^{*}(P)$ on $\mathcal{Y}$.

For a fixed point $b$ of any $\mathcal{E}_{i}$, we can choose local coordinates $\left(u_{1}, \ldots, u_{n-1}\right)$ around $b$ and local coordinates $\left(v_{1}, \ldots, v_{n-1}\right)$ around $\varphi(b)$. Then $\nu_{i}-1$ is defined to be the multiplicity of $\mathcal{E}_{i}$ in the local divisor defined by $\operatorname{det}\left(\frac{\partial\left(v_{1}, \ldots, v_{n-1}\right)}{\partial\left(u_{1}, \ldots, u_{n-1}\right)}\right)$. Remark that this is independent of the choice of $b$ on $\mathcal{E}_{i}$ and the choice of local coordinates around $b$ and $\varphi(b)$.

The ordered pairs of positive integers $\left(N_{i}, \nu_{i}\right), i \in \mathcal{T}$, are called the $n u$ merical data of the resolution $(\mathcal{Y}, \varphi)$. For $i \in \mathcal{T}$ and $I \subseteq \mathcal{T}$ we denote $\mathcal{E}_{i}^{\circ}:=\mathcal{E}_{i} \backslash \bigcup_{j \neq i} \mathcal{E}_{j}, \mathcal{E}_{I}:=\bigcap_{i \in I} \mathcal{E}_{i}$ and $\mathcal{E}_{I}^{\circ}:=\mathcal{E}_{I} \backslash \bigcup_{j \in \mathcal{T} \backslash I} \mathcal{E}_{j}$. In particular when $I=\emptyset$, we have that $\mathcal{E}_{\emptyset}=\mathcal{Y}$. Remark that $\mathcal{Y}$ is the disjoint union of the $\mathcal{E}_{I}^{\circ}$.

1.3. For any field extension $L$ of $F$ we can take the base extension of the resolution $(\mathcal{Y}, \varphi)$. The result will be an embedded resolution $\left(\mathcal{Y}^{L}, \varphi^{L}\right)$ of $\operatorname{Proj}\left(L\left[x_{1}, \ldots, x_{n}\right] /(f)\right)$ in Proj $L\left[x_{1}, \ldots, x_{n}\right]$ over $L$, with $\mathcal{Y}^{L}=\mathcal{Y}^{F} \times_{F} L$. If there is any danger of confusion we will include the field $L$ in the notation of (1.2) and thus write $\mathcal{P}^{L}, \mathcal{D}^{L}, \mathcal{E}_{i}^{L}, \mathcal{T}^{L}, \mathcal{T}_{e}^{L}, \mathrm{~T}_{s}^{L}, \mathcal{E}_{i}^{\circ L}, \mathcal{E}_{I}^{L}, \mathcal{E}_{I}^{\circ L}$.

For any field extension $L_{1} \subseteq L_{2}$ we have that $\mathcal{E}_{i}^{L_{1}} \times_{L_{1}} L_{2} \cong \bigcup_{j \in T_{i}} \mathcal{E}_{j}^{L_{2}}$, where $T_{i}$ is some subset of $\mathcal{T}^{L_{2}}$ and all $\mathcal{E}_{j}^{L_{2}}, j \in T_{i}$, have the same numerical data as $\mathcal{E}_{i}^{L_{1}}$. 
1.4. Now we can start our construction of a suitable embedded resolution of $f^{-1}\{0\}$ in $\mathbb{A}^{n}(F)$.

1.4.1. First consider the blowing-up $\pi: V \rightarrow \mathbb{A}^{n}(F)$ of $\mathbb{A}^{n}(F)$ with center the origin. Denote the strict transform of $f^{-1}\{0\}$ in $V$ with $\overline{f^{-1}\{0\}}$ and the inverse image of the origin (by $\pi$ ) with $E_{0}$. Remark that $E_{0} \cong \mathcal{P}$. Using the crucial ingredient that $f$ is homogeneous the following facts are not difficult to verify:

(i) the intersection $E_{0} \cap \overline{f^{-1}\{0\}}$ is isomorphic to $\mathcal{D}_{\text {red }}$;

(ii) there is an open covering $\left\{V_{i}\right\}$ of $E_{0}$ (respectively $\left\{U_{i}\right\}$ of $E_{0} \cap \overline{f-1\{0\}}$ ) such that $V$ (respectively $\overline{f^{-1}\{0\}}$ ) is obtained by glueing products of the form $\mathbb{A}^{1} \times V_{i}$ (respectively $\mathbb{A}^{1} \times U_{i}$ ).

1.4.2. Then we can find an embedded resolution $(Y, h)$ of $f^{-1}\{0\}$ in $\mathbb{A}^{n}(F)$ over $F$ by combining the point-centered blowing-up $\pi$ with the resolution of (1.2), taking into account the product with $\mathbb{A}^{1}$ pointed out in (ii) of (1.4.1). Let $E_{i}, i \in T=T_{e} \cup T_{s}$, be the (reduced) $F$-irreducible components of $h^{-1}\left(f^{-1}\{0\}\right)$, where $E_{i}$ is a (reduced) irreducible component of the exceptional divisor for $i \in T_{e}$ and of the strict transform of $f^{-1}\{0\}$ in $Y$ for $i \in T_{s}$. For each $i \in T$ let $N_{i}$ and $\nu_{i}-1$ be the multiplicities of $E_{i}$ in the divisor of respectively $f \circ h$ and $h^{*}\left(d x_{1} \wedge \cdots \wedge d x_{n}\right)$ on $Y$. The $\left(N_{i}, \nu_{i}\right)$, for $i \in T$, are called the numerical data of the resolution $(Y, h)$. For $i \in T$ and $I \subseteq T$ we denote $E_{i}^{\circ}:=E_{i} \backslash \bigcup_{j \neq i} E_{j}, E_{I}:=\bigcap_{i \in I} E_{i}$ and $E_{I}^{\circ}:=E_{I} \backslash \bigcup_{j \in T \backslash I} E_{j}$.

1.4.3. If $E_{0}$ also denotes the strict transform of $E_{0}$ in $Y$ (remark that $0 \in T_{e}$ in this situation), then the following remarks are easy consequences of the homogeneity of $f$ and the choice of our embedded resolution $(Y, h)$ :

(i) The intersection $E_{0} \cap E_{i}$ for $i \in T \backslash\{0\}$ is canonically isomorphic to one of the components $\mathcal{E}_{j}$ with $j \in \mathcal{T}$. This will give us a bijection between $T \backslash\{0\}$ and $\mathcal{T}$, so from now on we will assume $\mathcal{T}=T \backslash\{0\}$.

(ii) Under this identification of $\mathcal{T}$ with $T \backslash\{0\}$ also $\mathcal{T}_{e}$ and $\mathcal{T}_{s}$ coincide with respectively $T_{e} \backslash\{0\}$ and $T_{s}$, and corresponding $N_{i}$ and $\nu_{i}$ will be the same.

(iii) The second fact of (1.4.1) will also hold for $Y$ (respectively $E_{i}$ ) instead of $V$ (respectively $\overline{f^{-1}\{0\}}$ ).

(iv) The numerical data of $E_{0}$ are $N_{0}=\operatorname{deg} f$ and $\nu_{0}=n$; and $h^{-1}\{0\}=$ $E_{0}$.

1.4.4. In the same way as in (1.3) we can extend everything in (1.4) to any field extension $L$ of $F$.

1.4.5. Remark. From now on we associate to any homogeneous polynomial the embedded resolutions and other notations of (1.2)-(1.4). 
1.5. Now we are ready to state some general results in terms of the embedded resolutions constructed above. For the next two theorems we fix a homogeneous polynomial $f \in F\left[x_{1}, \ldots, x_{n}\right] \backslash F$, where $F$ is some number field.

Theorem 1.5.1 ([2, Section 3]). For almost all completions $K$ of $F$ (i.e., for all except a finite number) we have the following for a character $\kappa$ of $R_{K}^{\times}$ of order d.

(i) If $\kappa$ is not trivial on $1+P_{K}$, then $Z^{K}(\kappa, f, s)$ and $Z_{0}^{K}(\kappa, f, s)$ are constant on $\mathbb{C}$.

(ii) If $\kappa$ is trivial on $1+P_{K}$, then

$$
Z^{K}(\kappa, f, s)=q^{-n} \sum_{\substack{I \subseteq T^{K} \\ \forall i \in I: d \mid N_{i}}} C_{I, \kappa}^{K} \prod_{i \in I} \frac{q-1}{q^{\nu_{i}+s N_{i}}-1},
$$

with $C_{I, \kappa}^{K}=\sum_{k}(-1)^{k} \operatorname{Tr}\left[\right.$ Frob, $\left.H_{c}^{k}\left(\left(E_{I}^{\circ K}\right)_{\bar{K}}, \mathcal{L}_{\kappa}\right)\right]$.

Here $(\cdot)_{\bar{K}}$ denotes reduction modulo $P_{K}, \mathcal{L}_{\kappa}$ is a certain $\ell$-adic sheaf on $\left(Y^{K}\right)_{\bar{K}}$ associated to $\kappa$, $\operatorname{Tr}$ denotes the trace, and Frob is the geometric Frobenius of $\bar{K}$.

For $Z_{0}^{K}(\kappa, f, s)$ we have an analogous formula replacing $C_{I, \kappa}^{K}$ by a similar constant $C_{I, \kappa, 0}^{K}$.

(The explicit expression of $C_{I, \kappa}^{K}$ is just given for completeness; we will not need it in this paper.)

Theorem 1.5.2 ([5, Proposition 2 and Theorem 7]). For almost all completions $K$ of $F$ we have the following for a character $\kappa$ of $R_{K}^{\times}$of order $d$.

(i) If d does not divide $\operatorname{deg} f$, then $Z^{K}(\kappa, f, s)=Z_{0}^{K}(\kappa, f, s)=0$.

(ii) If $d$ divides $\operatorname{deg} f$ and $\kappa$ is trivial on $1+P_{K}$, then

$$
Z^{K}(\kappa, f, s)=\frac{\left(1-q^{-1}\right) q^{-(n-1)}}{1-q^{-(\operatorname{deg} f) s-n}} \sum_{\substack{I \subseteq \mathcal{T}^{K} \\ \forall i \in I: d \mid N_{i}}} \mathcal{C}_{I, \kappa}^{K} \prod_{i \in I} \frac{q-1}{q^{\nu_{i}+s N_{i}}-1}
$$

where $\mathcal{C}_{I, \kappa}^{K}$ is a certain constant.

1.5.3. Remark. By adapting the proof of Theorem 7 in [5] we easily find that under the same conditions as in (1.5.2)(ii)

$$
Z_{0}^{K}(\kappa, f, s)=q^{-(\operatorname{deg} f) s-n} \frac{\left(1-q^{-1}\right) q^{-(n-1)}}{1-q^{-(\operatorname{deg} f) s-n}} \sum_{\substack{I \subseteq \mathcal{T}^{K} \\ \forall i \in I: d \mid N_{i}}} \mathcal{C}_{I, \kappa}^{K} \prod_{i \in I} \frac{q-1}{q^{\nu_{i}+s N_{i}}-1}
$$


1.5.4. Remark. Although we stated the two theorems above only for a homogeneous polynomial, Theorem 1.5.1 also holds for an arbitrary polynomial. But Theorem 1.5.2 is specific for the homogeneous case.

1.6. We now remind the definition of local monodromy [9]. Fix $g \in$ $\mathbb{C}\left[x_{1}, \ldots, x_{n}\right] \backslash \mathbb{C}$ and $b \in \mathbb{C}^{n}$ with $g(b)=0$. Let $B \subseteq \mathbb{C}^{n}$ be a small enough ball with center $b$; the restriction $\left.g\right|_{B}$ is a locally trivial $C^{\infty}$ fibration over a small enough pointed disc $D \subseteq \mathbb{C} \backslash\{0\}$ with center 0 . Hence the diffeomorphism type of the Milnor fiber $M_{(g, b)}:=g^{-1}\{t\} \cap B$ of $g$ around $b$ does not depend on $t \in D$, and the counterclockwise generator of the fundamental group of $D$ induces an automorphism of $H^{\cdot}\left(M_{(g, b)}, \mathbb{C}\right)$ which is called the local monodromy of $g$ at $b$. By an eigenvalue of the local monodromy of $g$ at $b$ we mean an eigenvalue of the monodromy action on (at least) one of the $H^{q}\left(M_{(g, b)}, \mathbb{C}\right)$ for $q=0, \ldots, n-1$.

1.6.1. Remark. In the same way as in (1.4.2) we associate to an arbitrary embedded resolution of $g^{-1}\{0\}$ in $\mathbb{A}^{n}(\mathbb{C})$ the notations of that section. Now fix such an embedded resolution.

Theorem 1.6.2 ([1, Theorem 3]). For $b \in g^{-1}\{0\}$ let $P_{q}(t)$ denote the characteristic polynomial of the monodromy action on $H^{q}\left(M_{(g, b)}, \mathbb{C}\right)$ for $q=$ $0, \ldots, n-1$. Then

$$
\prod_{q=0}^{n-1}\left(P_{q}(t)\right)^{(-1)^{q+1}}=\prod_{i \in T}\left(1-t^{N_{i}}\right)^{-\chi\left(E_{i}^{\circ} \cap h^{-1}\{b\}\right) .}
$$

1.6.3. In particular if $b$ is the origin and if $g$ is homogeneous, then (1.4.3)(iv) implies that $\prod_{q=0}^{n-1}\left(P_{q}(t)\right)^{(-1)^{q+1}}=\left(1-t^{\operatorname{deg} g}\right)^{-\chi\left(E_{0}^{\circ}\right)}$. This assertion is also classically known and follows for example from [9, Section 9].

\section{Holomorphy conjecture for homogeneous polynomials.}

2.1. In this section we will use the embedded resolutions of Section 1 to provide in arbitrary dimension an easy proof of the holomorphy conjecture for homogeneous polynomials under the additional characteristic-assumption mentioned before in the introduction. In the next section we will treat the case in which this assumption is not fulfilled.

Theorem 2.2. Let $F$ be a number field and $f \in F\left[x_{1}, \ldots, x_{n}\right] \backslash F$ a homogeneous polynomial such that $\chi\left(\mathbb{P}_{\mathbb{C}}^{n-1} \backslash\{f=0\}\right) \neq 0$. For almost all completions $K$ of $F$ we have the following for any character $\kappa$ of $R_{K}^{\times}$. If the order of $\kappa$ does not divide the order of any eigenvalue of the (complex) local monodromy of $f$ at any point of $f^{-1}\{0\}$, then $Z^{K}(\kappa, f, s)$ and $Z_{0}^{K}(\kappa, f, s)$ are holomorphic on $\mathbb{C}$. In fact they are identically zero. 
Proof. We use the construction and notation of (1.2)-(1.4). It follows from this construction (especially from $(1.4 .1)(i))$ that $\chi\left(E_{0}^{\circ}\right)=\chi\left(\mathbb{P}_{\mathbb{C}}^{n-1} \backslash\{f=\right.$ $0\}) \neq 0$. So (1.6.3) implies that $e^{\frac{2 \pi i}{\operatorname{deg} f}}$ is an eigenvalue of the local monodromy of $f$ at the origin. From the conditions in the holomorphy conjecture we find that the order of the character $\kappa$ does not divide $\operatorname{deg} f$, which gives us the result by Theorem 1.5.2(i).

2.3. Now we introduce the so-called topological zeta function $Z_{\text {top }}^{(r)}(g, s)$, which is associated to $g \in \mathbb{C}\left[x_{1}, \ldots, x_{n}\right]$ and $r \in \mathbb{N} \backslash\{0\}$ by Denef and Loeser [4, Section 3]. With the notations from remark (1.6.1) we have that

$$
Z_{\mathrm{top}}^{(r)}(g, s)=\sum_{\substack{I \subseteq T \\ \forall i \in I: r \mid N_{i}}} \chi\left(E_{I}^{\circ}\right) \prod_{i \in I} \frac{1}{\nu_{i}+N_{i} s},
$$

with $s \in \mathbb{C}$. Replacing $\chi\left(E_{I}^{\circ}\right)$ by $\chi\left(E_{I}^{\circ} \cap h^{-1}\{0\}\right)$ we analogously define $Z_{\text {top }, 0}^{(r)}(g, s)$. When $g$ is homogeneous it will easily follow from the proof of Theorem 2.4 that $Z_{\text {top }}^{(r)}(g, s)=Z_{\text {top }, 0}^{(r)}(g, s)$. So from now on we just have to deal with one of them. We can also formulate a holomorphy conjecture for this topological zeta function.

Conjecture 2.3.1. If $r \in \mathbb{N} \backslash\{0\}$ does not divide the order of any eigenvalue of the local monodromy of $g$ at any point of $g^{-1}\{0\}$, then $Z_{\mathrm{top}}^{(r)}(g, s)$ is holomorphic on $\mathbb{C}$.

Theorem 2.4. Let $f \in \mathbb{C}\left[x_{1}, \ldots, x_{n}\right] \backslash \mathbb{C}$ be a homogeneous polynomial, with $\chi\left(\mathbb{P}_{\mathbb{C}}^{n-1} \backslash\{f=0\}\right) \neq 0$. Then the holomorphy conjecture is true for $Z_{\text {top }}^{(r)}(f, s)$.

Proof. Let $r \in \mathbb{N} \backslash\{0\}$ such that $r$ does not divide the order of any eigenvalue of the local monodromy of $f$ at any point of $f^{-1}\{0\}$. As in the previous proof we find that $r$ does not divide $\operatorname{deg} f$, so with the notations of Section 1 the topological zeta function reduces to

$$
Z_{\text {top }}^{(r)}(f, s)=\sum_{\substack{0 \notin I \subseteq T \\ \forall i \in I: r \mid N_{i}}} \chi\left(E_{I}^{\circ}\right) \prod_{i \in I} \frac{1}{\nu_{i}+N_{i} s} .
$$

Now consider a subset $I$ of $T$ with $0 \notin I$ and $\forall i \in I: r \mid N_{i}$. From (1.4.3)(iii) we know that (locally) $E_{I}^{\circ}=H_{I} \times\left(\mathbb{A}^{1} \backslash\{\right.$ point $\left.\}\right)$, where $H_{I}$ is some subvariety of $E_{0}$. Because $\chi\left(\mathbb{A}^{1} \backslash\{\right.$ point $\left.\}\right)=0$, we can easily conclude that $\chi\left(E_{I}^{\circ}\right)=0$, implying $Z_{\text {top }}^{(r)}(f, s)$ to be zero. 


\section{The case of Euler-Poincaré characteristic zero.}

3.1. When $\chi\left(\mathbb{P}_{\mathbb{C}}^{n-1} \backslash\{f=0\}\right)=0$ for the homogeneous polynomial $f$ in question, we are still able to prove the holomorphy conjecture by assuming a sort of 'projective holomorphy conjecture in $\mathbb{P}^{n-1}$. For $n=3$ this will actually give us a tool to prove the holomorphy conjecture by using the fact that the holomorphy conjecture is true for curves. (See $[\mathbf{1 0}]$.)

3.2. Suppose that $K$ is the completion of a number field $F$ with respect to some maximal ideal of its ring of integers and that $\kappa$ is a character of $R_{K}^{\times}$ of order $d$ such that $d$ divides $\operatorname{deg} f$ and $\kappa$ is trivial on $1+P_{K}$. We define the projective local zeta function associated to $f$ and $\kappa$ to be

$$
Z_{\text {proj }}^{K}(\kappa, f, s)=\frac{1-q^{-(\operatorname{deg} f) s-n}}{1-q^{-1}} Z^{K}(\kappa, f, s) .
$$

By Theorem 1.5.2(ii) we have, using the notations of Section 1, that

$$
Z_{\text {proj }}^{K}(\kappa, f, s)=q^{-(n-1)} \sum_{\substack{I \subseteq \mathcal{T}^{K} \\ \forall i \in I: d \mid N_{i}}} \mathcal{C}_{I, \kappa}^{K} \prod_{i \in I} \frac{q-1}{q^{\nu_{i}+s N_{i}}-1},
$$

for almost all fields $K$ and all characters $\kappa$ as above. By comparing this expression with Theorem 1.5.1(ii) the underlying inspiration for this definition should be clear.

3.3. Under the holomorphy conjecture for $Z_{\text {proj }}^{K}(\kappa, f, s)$ we understand: For almost all fields $K$ and for all characters $\kappa$ as in (3.2) we have the following. If the order of $\kappa$ does not divide the order of any eigenvalue of the (complex) local monodromy of any $f_{j}$ at any complex point of $f_{j}^{-1}\{0\}$, then $Z_{\text {proj }}^{K}(\kappa, f, s)$ is holomorphic on $\mathbb{C}$.

Here $f_{j}$ denotes the polynomial you get by putting $x_{j}=1$ in the homogeneous polynomial $f$. Before stating the next theorem we formulate a lemma that we will need in its proof.

Lemma 3.4 ([3, proof of Proposition 3.4]). Let $g \in \mathbb{C}\left[x_{1}, \ldots, x_{n}\right] \backslash \mathbb{C}$. If $\lambda$ is an eigenvalue of the local monodromy of $g$ at some point $b$ on $g^{-1}\{0\}$, then there is a point $c$ on $g^{-1}\{0\}$ such that $\lambda$ is a zero or a pole of the alternating product of the characteristic polynomials of the monodromy action on $H^{i}\left(M_{(g, c)}, \mathbb{C}\right)$ for $i=0, \ldots, n-1$.

Theorem 3.5. Let $F$ be a number field and $f \in F\left[x_{1}, \ldots, x_{n}\right] \backslash F$ a homogeneous polynomial. The holomorphy conjecture for $Z_{\mathrm{proj}}^{K}(\kappa, f, s)$ implies the holomorphy conjecture for $Z^{K}(\kappa, f, s)$ (and for $\left.Z_{0}^{K}(\kappa, f, s)\right)$. 
Remark. Of course this theorem holds when $\chi\left(\mathbb{P}_{\mathbb{C}}^{n-1} \backslash\{f=0\}\right) \neq 0$, but we know from the proof of Theorem 2.2 that this condition implies that the order of $\kappa$ does not divide $\operatorname{deg} f$. So in the light of Theorem 1.5.2(i), we only have to deal with a nontrivial case if $\chi\left(\mathbb{P}_{\mathbb{C}}^{n-1} \backslash\{f=0\}\right)=0$.

Proof of Theorem 3.5. By Theorem 1.5.1(i) and Theorem 1.5.2(i) we may assume that $\kappa$ is trivial on $1+P_{K}$ and that the order $d$ of $\kappa \operatorname{divides} \operatorname{deg} f$. So we let $K$ and $\kappa$ be as in (3.2).

Suppose that the order of $\kappa$ divides the order of some eigenvalue $\lambda$ of the local monodromy of some $f_{j}$ at some point $b$ of $f_{j}^{-1}\{0\}$. Then by Lemma 3.4 we may assume that $\lambda$ is a zero or a pole of the alternating product of the characteristic polynomials of the monodromy action on $H^{i}\left(M_{\left(f_{j}, b\right)}, \mathbb{C}\right)$ for $i=0, \ldots, n-2$. Now, using Theorem 1.6.2 and the construction in (1.4) (especially the results of (1.4.3)), it is not hard to find a point $c$ (different from the origin) of $f^{-1}\{0\}$ such that the product above equals the alternating product of the characteristic polynomials of the monodromy action on $H^{i}\left(M_{(f, c)}, \mathbb{C}\right)$ for $i=0, \ldots, n-1$. So $\lambda$ will also be an eigenvalue of the local monodromy of $f$ at $c$.

We can conclude that the condition in the holomorphy conjecture (for $Z^{K}(\kappa, f, s)$ and $\left.Z_{0}^{K}(\kappa, f, s)\right)$ implies the condition in the holomorphy conjecture for $Z_{\text {proj }}^{K}(\kappa, f, s)$, and hence by assumption that $Z_{\text {proj }}^{K}(\kappa, f, s)$ is holomorphic on $\mathbb{C}$ (for almost all completions $K$ of $F$ ). Because $Z_{\text {proj }}^{K}(\kappa, f, s)$ is a rational function in $q^{-s}$ (of non-positive degree), this yields that $Z_{\text {proj }}^{K}(\kappa, f, s)$ is constant as function of $s$, and more concretely

$$
Z_{\text {proj }}^{K}(\kappa, f, s)=\frac{1}{q^{n-1}} \mathcal{C}_{\emptyset, \kappa}^{K} .
$$

From (1.5.3) and (3.2) we find that

$$
\begin{aligned}
Z_{0}^{K}(\kappa, f, s) & =q^{-(\operatorname{deg} f) s-n} Z^{K}(\kappa, f, s) \\
& =\frac{\left(1-q^{-1}\right) q^{-(\operatorname{deg} f) s-n}}{1-q^{-(\operatorname{deg} f) s-n}} \frac{1}{q^{n-1}} \mathcal{C}_{\emptyset, \kappa}^{K},
\end{aligned}
$$

which is of degree zero (as rational function in $q^{-s}$ ) if $\mathcal{C}_{\emptyset, \kappa}^{K}$ is different from zero. But since Theorem 1.2 in [3] says that for almost all completions $K$ of $F$ the degree of $Z_{0}^{K}(\kappa, f, s)$ has to be strictly negative, we can conclude that $\mathcal{C}_{\emptyset, \kappa}^{K}=0$ and so that $Z^{K}(\kappa, f, s)=0$ (and of course also that $Z_{0}^{K}(\kappa, f, s)=$ $0)$. 
3.6. Topological zeta function. Let $f \in \mathbb{C}\left[x_{1}, \ldots, x_{n}\right] \backslash \mathbb{C}$ be a homogeneous polynomial and $r \in \mathbb{N} \backslash\{0\}$ such that $r$ divides $\operatorname{deg} f$. Looking at the definition of $Z_{\text {top }}^{(r)}(f, s)$, but now thinking projectively, we are stimulated to define

$$
Z_{\text {top,proj }}^{(r)}(f, s):=\sum_{\substack{I \subseteq \mathcal{T} \\ \forall i \in I: r \mid N_{i}}} \chi\left(\mathcal{E}_{I}^{\circ}\right) \prod_{i \in I} \frac{1}{\nu_{i}+s N_{i}} .
$$

Then by the proof of Theorem 2.4 and by the facts of (1.4.3) we see that $Z_{\text {top }}^{(r)}(f, s)=\frac{1}{n+(\operatorname{deg} f) s} Z_{\text {top,proj }}^{(r)}(f, s)$, so obviously $Z_{\text {top,proj }}^{(r)}(f, s)$ is independent of the chosen embedded resolution of $\mathcal{D}$ in $\mathcal{P}$ as in (1.2).

3.6.1. The holomorphy conjecture for $Z_{\text {top,proj }}^{(r)}(f, s)$ can be formulated as follows. If $r \in \mathbb{N} \backslash\{0\}$ does not divide the order of any eigenvalue of the local monodromy of any $f_{j}$ at any point of $f_{j}^{-1}\{0\}$ and if $r \mid \operatorname{deg} f$, then $Z_{\text {top,proj }}^{(r)}(f, s)$ is holomorphic on $\mathbb{C}$.

3.6.2. Under the condition $\chi\left(\mathbb{P}_{\mathbb{C}}^{n-1} \backslash\{f=0\}\right)=0$ the analogue of Theorem 3.5 will hold for the topological zeta function. Indeed, if $r \nmid \operatorname{deg} f$, then $Z_{\text {top }}^{(r)}(f, s)$ is holomorphic on $\mathbb{C}$ by the proof of Theorem 2.4. If $r \mid \operatorname{deg} f$, then the same idea as in the proof of Theorem 3.5 will give us (under the assumption of the holomorphy conjecture for $\left.Z_{\text {top,proj }}^{(r)}(f, s)\right)$ that $Z_{\text {top }}^{(r)}(f, s)=$ $\frac{1}{n+(\operatorname{deg} f) s} \chi\left(\mathbb{P}_{\mathbb{C}}^{n-1} \backslash\{f=0\}\right)=0$.

Theorem 3.7. For a homogeneous polynomial $f$ in three variables the holomorphy conjectures for Igusa's local zeta functions $Z^{K}(\kappa, f, s)$ and $Z_{0}^{K}(\kappa, f, s)$ and for the topological zeta function $Z_{\text {top }}^{(r)}(f, s)$ are true.

Proof. From the previous results it is clear that we just have to prove the holomorphy conjecture for $Z_{\text {proj }}^{K}(\kappa, f, s)$ (and $Z_{\text {top,proj }}^{(r)}(f, s)$ ).

Take for the embedded resolution $\left(\mathcal{Y}^{F}, \varphi^{F}\right)$ of (1.2) the (scheme-theoretical) canonical embedded resolution of $\mathcal{D}^{F}$ in $\mathcal{P}^{F}$. By restricting we get the canonical embedded resolution of $f_{j}^{-1}\{0\}$ in $\mathbb{A}^{2}(F)$ for any $j \in\{1,2,3\}$, so we can use on every affine chart the same arguments as in the proof of the holomorphy conjecture for curves [10]. By noting that the number $N_{i}$ of an irreducible component of the strict transform does not change by passing to an affine chart, the proof of Theorem 3.7 is done.

\section{The monodromy conjecture.}

4.1. Under the usual characteristic-assumption we will show in this section how to prove the so-called monodromy conjecture of Igusa for an homogeneous polynomial in three variables using mainly the same ideas as in the proof of Theorem 3.7. 
Theorem 4.2. Let $F$ be a number field and $f \in F\left[x_{1}, x_{2}, x_{3}\right] \backslash F$ a homogeneous polynomial such that $\chi\left(\mathbb{P}_{\mathbb{C}}^{2} \backslash\{f=0\}\right) \neq 0$. For almost all completions $K$ of $F$ we have the following for any character $\kappa$ of $R_{K}^{\times}$. If $s_{0}$ is a pole of $Z^{K}(\kappa, f, s)$, then $\exp \left(2 \pi \sqrt{-1} \Re\left(s_{0}\right)\right)$ is an eigenvalue of the local monodromy of $f$ at some complex point of $f^{-1}\{0\}$.

Proof. As in the proof of Theorem 3.7 we take for the embedded resolution $\left(\mathcal{Y}^{F}, \varphi^{F}\right)$ of (1.2) the (scheme-theoretical) canonical embedded resolution of $\mathcal{D}^{F}$ in $\mathcal{P}^{F}$. By Theorem 1.5.1(i) and Theorem 1.5.2(ii) we may assume that $Z^{K}(\kappa, f, s)=\frac{1-q^{-1}}{1-q^{-(\operatorname{deg} f) s-3}} Z_{\text {proj }}^{K}(\kappa, f, s)$. Because $\exp \left(2 \pi i\left(\frac{-3}{\operatorname{deg} f}\right)\right)$ is an eigenvalue of the local monodromy of $f$ at the origin (see the proof of Theorem 2.2), we may also assume that $s_{0}$ is a pole of $Z_{\mathrm{proj}}^{K}(\kappa, f, s)$. As in the proof of Theorem 5.2.1 in [2], we find that $\Re\left(s_{0}\right)=\frac{-\nu_{j}}{N_{j}}$ for some $j \in \mathcal{T}^{\mathbb{C}}$, with $\left|\mathcal{E}_{j}^{\mathbb{C}} \backslash \mathcal{E}_{j}^{\circ \mathbb{C}}\right| \geq 3$ or $j \in \mathcal{T}_{s}^{\mathbb{C}}$. First suppose that $j \in \mathcal{T}_{s}^{\mathbb{C}}$. Then for some $k \in\{1,2,3\}$ there is an irreducible component of $f_{k}^{-1}\{0\}$ in $\mathbb{A}^{2}(\mathbb{C})$, whose strict transform (by the restriction of $\varphi^{\mathbb{C}}$ ) has numerical data $\left(N_{j}, \nu_{j}\right)=\left(N_{j}, 1\right)$. It is well-known that in this case $\exp \left(2 \pi i\left(\frac{-\nu_{j}}{N_{j}}\right)\right)$ is an eigenvalue of the local monodromy of $f_{k}$ at some nonsingular point of $f_{k}^{-1}\{0\}$. As in the proof of Theorem 3.5 this implies that $\exp \left(2 \pi i \Re\left(s_{0}\right)\right)$ is an eigenvalue of the local monodromy of $f$ at some complex point of $f^{-1}\{0\}$. Next suppose that $j \notin \mathcal{T}_{s}^{\mathbb{C}}$ and $\left|\mathcal{E}_{j}^{\mathbb{C}} \backslash \mathcal{E}_{j}^{\circ \mathbb{C}}\right| \geq 3$. Then $\mathcal{E}_{j}^{\mathbb{C}}$ will also be an exceptional curve of the restriction of $\varphi^{\mathbb{C}}$ to some affine chart, which is in fact the canonical embedded resolution of $f_{k}^{-1}\{0\}$ in $\mathbb{A}^{2}(\mathbb{C})$ for some $k \in\{1,2,3\}$. Now it is known, see for example [8], that then $\exp \left(2 \pi i \Re\left(s_{0}\right)\right)$ is an eigenvalue of the local monodromy of $f_{k}$ at some point of $f_{k}^{-1}\{0\}$. Then again we can conclude that $\exp \left(2 \pi i \Re\left(s_{0}\right)\right)$ is also an eigenvalue of the local monodromy of $f$ at some complex point of $f^{-1}\{0\}$. This completes the proof of Theorem 4.2 .

4.2.1. Remark. From Remark 1.5 .3 we know that

$$
Z_{0}^{K}(\kappa, f, s)=q^{-(\operatorname{deg} f) s-n} Z^{K}(\kappa, f, s),
$$

so the monodromy conjecture is clearly also true for $Z_{0}^{K}(\kappa, f, s)$ when $\chi\left(\mathbb{P}_{\mathbb{C}}^{2} \backslash\right.$ $\{f=0\}) \neq 0$.

4.2.2. Remark. In a similar way we can prove the monodromy conjecture for the topological zeta function (again for a homogeneous polynomial $f$ in three variables and under the condition that $\left.\chi\left(\mathbb{P}_{\mathbb{C}}^{2} \backslash\{f=0\}\right) \neq 0\right)$. This conjecture states the following for $r \in \mathbb{N} \backslash\{0\}$. If $s_{0}$ is a pole of $Z_{\mathrm{top}}^{(r)}(f, s)$, then $\exp \left(2 \pi \sqrt{-1} s_{0}\right)$ is an eigenvalue of the local monodromy of $f$ at some complex point of $f^{-1}\{0\}$. 


\section{References}

[1] N. A'Campo, La fonction zeta d'une monodromie, Comment. Math. Helv., 50 (1975), 233-248, MR 51 \#8106, Zbl 333.14008.

[2] J. Denef, Report on Igusa's local zeta function, Sém. Bourbaki, 201/202/203 (1991), 359-386, MR 93g:11119, Zbl 749.11054.

[3] _ Degree of local zeta functions and monodromy, Compositio Math., 89 (1993), 207-216, MR 95g:11118, Zbl 932.11073.

[4] J. Denef and F. Loeser, Caractéristiques d'Euler-Poincaré, fonctions zeta locales, et modifications analytiques, J. Amer. Math. Soc., 5 (1992), 705-720, MR 93g:11118, Zbl 777.32017 .

[5] J. Denef and D. Meuser, A functional equation of Igusa's local zeta function, Amer. J. Math., 113 (1991), 1135-1152, MR 93e:11145, Zbl 749.11053.

[6] H. Hironaka, Resolution of singularities of an algebraic variety over a field of characteristic zero, Ann. Math., 79 (1964), 109-326, MR 33 \#7333, Zbl 122.38603.

[7] J. Igusa, Complex powers and asymptotic expansions I, J. Reine Angew. Math., 268/269 (1974), 110-130; II, ibid., 278/279 (1975), 307-321, MR 53 \#8018, Zbl 315.41029.

[8] F. Loeser, Fonctions d'Igusa p-adiques et polynômes de Bernstein, Amer. J. Math., 110 (1988), 1-22, MR 89d:11110, Zbl 644.12007.

[9] J. Milnor, Singular points of complex hypersurfaces, Princeton Univ. Press, 1968, MR 39 \#969, Zbl 184.48405.

[10] W. Veys, Holomorphy of local zeta functions for curves, Math. Ann., 295 (1993), 635-641, MR 94c:11115, Zbl 786.11068.

Received February 1, 2000 and revised November 8, 2000. The first author is a Research Assistant of the Belgian Fund for Scientific Research - Flanders.

Department of Mathematics

UNIVERSITY OF LEUVEN

Celestijnenlaan 200B

B-3001 Leuven

BELGiUM

E-mail address: bart.rodrigues@wis.kuleuven.ac.be

Department of Mathematics

UNIVERSITY OF LEUVEN

Celestijnenlaan 200B

B-3001 LEUVEN

BELGIUM

E-mail address: wim.veys@wis.kuleuven.ac.be 